\title{
Graphene-Based Biosensors for Nano and Pico Applications: The Future is here!
}

\section{Farid Menaa*}

Department of Pharmaceutical Sciences and Nano-Medicine Fluorotronics, Inc. San Diego, California, USA

Keywords: Graphene; Spectroscopy; Biosensing; Personalized medicine; Oncology

Nanotechnology and smaller scale technologies represent golden eggs for medicine and sciences [1,2]. In the recent years, graphene (G)-based biosensors, including functional graphene oxide (GO) and $G$ hybrid nanocomposites, are increasingly explored for real-time imaging and quantification of biomolecules or cells [3]. The remarkable intrinsic and tunable properties of $\mathrm{G}$ and derivatives (e.g. planar structure, high surface-volume ratio, high electrical conductivity, good chemical stability and strong mechanical strength) are quite attracting to manufacture reliable, highly sensitive and ultra-fast biosensing platforms (e.g. label-free or fluorochrome-based nano-optical/ biophotonic detection systems such as FRET or CRET) [3]. A number of emerging studies have reported a combination of functional, green, cost-effective and scalable approaches to constantly improve the overall properties (e.g. sensitivity, specificity/selectivity, stability, rapidity, reproducibility) of the $\mathrm{G}$ component for real-time and multiplexed imaging of biomolecules (e.g. biomarkers of disease, nucleic acid alterations) or cells (e.g. cancer cells, stem cells, bacteria or viruses) [3]. G and derivatives-based biosensors, which besides can be used as nano-vehicles for drug delivery [2], are revolutionizing the disease prognosis, diagnosis and therapy [3]. The possibility to detect and characterize a single cell or very lowly expressed biomolecules makes G-derived biosensors among the most promising tools for efficient translational, integrative, regenerative and personalized medicine $[3,4]$. However, although the cell viability in vitro is not affected, the potential nanocytoxicity (i.e. nanocompatibility and consequences of uncontrolled nanobiogradability) in a clinical setting using humans remains unknown. Eventually, the establishment and compliance of international regulatory industrial and healthcare guidelines before commercialization and translational medicine are still requested. Owing to the possible synergic or additive effects of fluorine and graphenebased materials for efficient personalized medicine [5-7], fluorinated-G nanosystems are emerging and might overcome many limitations encountered with a number of pharmaceutical and medical applications (e.g. quantitative determination of very low concentrations of biological or chemicals; stem cells growth and differentiation; controlled tumorsite targeted drug delivery). Indeed, these G-nanosystems can be characterized and monitored by state-of-the art technologies such as carbon-fluorine spectroscopy (aka SpectroFluor ${ }^{\mathrm{TM}}$ ) platform [7].

Overall, the recent advances of G-based biosensors are quite important for translational, personalized, integrative, regenerative, and also green medicine. G-based biosensing is strived to a bright future, bringing new hopes for the diagnosis and prognosis of patients, while offering to the healthcare professional the possibility to use alternative therapeutic options.

\section{References}

1. Menaa B (2011) The importance of Nanotechnology in Biomedical Sciences. J Biotechnol Biomaterial 1:105e.

2. Menaa F (2013) When Pharma Meets Nano or The Emerging Era of NanoPharmaceuticals. Pharmaceut Anal Acta 4: 223.

3. Menaa F (2013) Functional Graphene-Based Nanobioimaging Platforms: New Powered Real-Time Interfaces. J Mol Imaging Dynam 2:e103.

4. Menaa F (2013) 2-D Graphene and Derivatives-Based Scaffolds in Regenerative Medicine: Innovative Mimicking 3-D Cell Microenvironment. J Regen Med 2: 2.

5. Menaa F, Menaa B, Sharts ON (2013) Importance of Fluorine and Fluorocarbons in Medicinal Chemistry and Oncology. J Mol Pharm Org Process Res 1:104.

6. Menaa F, Menaa B, Kundu PP, Narayana C, Sharts ON (2013) Physical Characterization of Blood Substitutes by Carbon-Fluorine Spectroscopy. Pharmaceut Anal Acta 4: 235

7. Menaa F, Menaa B, Sharts O (2011) Development of carbon-fluorine spectroscopy for pharmaceutical and biomedical applications. Faraday Discuss 149: $269-278$.
*Corresponding author: Farid Menaa, Director, Department of Pharmaceutical Sciences and Nano-Medicine Fluorotronics, Inc. San Diego, California, USA, Tel: (+1) 858-274-2728; E-mail: dr.fmenaa@gmail.com

Received December 04, 2013; Accepted December 12, 2013; Published December 13, 2013

Citation: Menaa F (2013) Graphene-Based Biosensors for Nano and Pico Applications: The Future is here! Pharm Anal Acta 5: e161 doi: 10.4172/21532435.1000e161

Copyright: () 2013 Menaa F. This is an open-access article distributed under the terms of the Creative Commons Attribution License, which permits unrestricted use, distribution, and reproduction in any medium, provided the original author and source are credited. 